\title{
Identification of a functional p53 responsive element within the promoter of XAF1 gene in gastrointestinal cancer cells
}

\author{
WENJING ZHANG, ZHENG GUO, BO JIANG, LINGYUN NIU, GUOSHENG XIA, \\ XINYING WANG, TIANMING CHENG, YUSHENG ZHANG and JIDE WANG \\ Department of Digestive Medicine, Nanfang Hospital, The Southern Medical University, \\ Guangzhou 510515, P.R. China
}

Received October 16, 2009; Accepted January 7, 2010

DOI: 10.3892/ijo_00000584

\begin{abstract}
It has been reported that XAF1 expression in gastric cancer is negatively correlated with p53. Our purpose was to clarify the regulatory mechanism of p53 on XAF1 expression. The effects of overexpressed wild-type and mutant p53 on XAF1 expression were evaluated. Binding capacity of core $\mathrm{XAF} 1$ promoter sequence to the recombinant $\mathrm{p} 53$ protein was examined. Site-directed mutation of putative p53 binding sequence and p53 knockdown by siRNA were performed. The protein expression and promoter activities of XAF1 in cells with null p53 were higher than that with wild-type and mutant p53. Ectopic overexpression of wild-type p53 suppressed XAF1 expression. A half-site (-95 to $-86 \mathrm{nt})$ and a quarter-site $(-4$ to $+1 \mathrm{nt})$ of $\mathrm{p} 53$ responsive element were found within XAF1 promoter. Both sequences bound to recombinant p53 effectively and specifically. Site-mutation of p53 responsive sequences abrogated the binding capacity. However, only the mutation of half-site increased XAF1 promoter activities. Suppression of p53 not only decreased the binding capacity of p53 responsive halfsite but also increased XAF1 transcription. In conclusion, we demonstrated that p53 could suppress the transcription of XAF1 through interaction with a high affinity responsive element (-95 to -86 nt) within XAF1 promoter, indicating a novel exclusive mechanism between these two tumor suppressors.
\end{abstract}

\section{Introduction}

p53 is a tumor suppressor that is involved in the regulation of various cellular events, such as DNA damage and repair,

Correspondence to: Dr Jide Wang, Department of Digestive Medicine, Nanfang Hospital, The Southern Medical University, Guangzhou 510515, P.R. China

E-mail: jidewang@gmail.com

Abbreviations: GI, gastrointestinal; PFT, pifithrin $\alpha$; EMSA, electrophoretic mobility shift assay; RLU, relative luciferase unit; p53RE, p53 responsive element

Key words: p53, XAF1, transcription regulation, gastrointestine, cancer hypoxia stress, cell division and cell cycle progression $(1,2)$. As a transcription factor, at least 129 target genes have been identified to be either activated or repressed by p53 (3). The functions of these genes covered almost all regulatory pathways related to cell growth control. Loss of the proper p53 expression by either gene mutation or deletion has been considered one of the major tumorigenetic mechanisms for most of cancers including gastrointestinal (GI) cancers (4).

p53 modulates the transcription of its target genes mainly through the direct binding to the sequence specific responsive element within their promoters. Unlike most other transcription factors whose binding sequence is relatively unique, the binding element of p53 is degenerate with some uncertainty $(3,5,6)$. The consensus sequence is composed of 5'-RRRCWWGYYY-3', where $\mathrm{R}$ is a purine, $\mathrm{Y}$ is a pyrimidine, $\mathrm{W}$ is either A or T (adenine or thymine), $\mathrm{G}$ is guanine and $\mathrm{C}$ is cytosine. The p53-binding site in the genome of many organisms is composed of a half-site RRRCWWGYYY followed by a spacer, usually composed of 0-21 base pairs, which is then followed by a second half-site RRRCWWGYYY sequence $(3,6)$. In addition, one half-site can be further divided into two quarter-sites RRRCW and WGYYY. The assembly mode of this couple of quarter-sites in different orientation can all bind to p53 with the same affinity $(3,6)$. Traditionally, it is believed that all four quarter-sites are required for the direct binding of p53 to DNA as active p53 exists in a form of tetramer and each p53 subunit binds to one quarter-site in its consensus sequence $(7,8)$. However, two recent studies demonstrated that $\mathrm{p} 53$ tetramer could use only two monomers to recognize DNA sequence and induce DNA bending $(9,10)$. Moreover, p53 can also regulate its target gene indirectly by acting as a transactivator to other DNAbinding protein such as TATA-binding protein (TBP) (11). p53 can even activate the transcription of the c-erbA- $\alpha$ gene through a DNA element that contains only one p53 quartersite in the presence of GC3 element (12).

XAF1 (XIAP-associated factor 1) was first defined as a XIAP interacting protein that antagonized the effect of XIAP in inhibiting caspases activation (13). Thus, XAF1 was implicated as a tumor suppressor. Fluorescence in situ hybridization analysis localized the XAF1 gene locus at 17p13.2, telomeric to the p53 gene (14). The XAF1 locus was further refined to YAC $746 \mathrm{C} 10$, approximately $3 \mathrm{cM}$ distal to Tp53 (14). The expression of XAF1 in GI cancers is 
lower than that of normal tissues due to promoter methylation in cancer cells $(15,16)$. Interestingly, Byun et al reported that XAF1 gene silencing in gastric cancer cells is negatively correlated with the mutation or deletion of $\mathrm{p} 53$ with the regulatory mechanisms unknown (15).

In this study, we first confirmed the negative correlation between XAF1 expression and p53 and then identified a halfsite p53 binding sequence with high affinity with the recombinant p53 protein. Both the site-mutation of this binding sequence and suppression of the p53 expression by siRNA could upregulate XAF1 transcription. Our result defined XAF1 as a novel p53 target gene.

\section{Materials and methods}

Cell lines and reagent. Human gastric and colon cancer cell lines AGS, Kato-III, HCT116, LoVo, SW480, SW1116, and HCT15 were all obtained from ATCC (Rockville, MD, USA) and cultured as described previously $(16,17)$. Pifithrin- $\alpha$ (PFT) was purchased from Sigma (St. Louis, MO, USA). Recombinant p53 protein, p53 gel shift oligonucleotides (double strand p53 consensus oligonucleotides), goat antihuman XAF1 (C-16), actin (I-19), mouse anti-human p53 (Pab1801), HRP-conjugated anti-goat IgG and anti-mouse IgG were all purchased from Santa Cruz Biotechnology (Santa Cruz, CA, USA).

Constructs and transient transfection. Luciferase reporter construct of XAF1 promoter pLuc-107 that contained the $107 \mathrm{bp}$ XAF1 promoter segment upstream of the ATG translation starting codon has been described before $(16,17)$. pCMV-p53 and pCMV-p53mt135 expressing wild-type and dominant-negative $\mathrm{p} 53$ proteins, respectively, were purchased from clontech (Mountain View, CA, USA). Empty vector pCMV-tag1 was the product of Stratagene (La Jolla, CA, USA). Transient transfection was carried out using Lipofectamine 2000 reagent according to the protocol of the manufacturer. For sequential transfection of p53 expressing vectors with luciferase reporter construct, the cells were transiently transfected with empty vector or p53 vectors followed by selection with medium containing $1000 \mu \mathrm{g} / \mathrm{ml}$ of G418 for 1 week to enrich the transfected cell clones followed by transfection with the luciferase reporter vector.

siRNA transfection. The siRNA duplexes consisted of 21 base pairs with a 2-base deoxynucleotide overhang (Proligo, Singapore). The sequences of the p53 and control siRNA (GL2, against the renilla luciferase gene) have been reported before and were as follows (sense strand): p53 siRNA: 5'-C UACUUCCUGAAAACAACGTT-3', GL2 siRNA, 5'-CGU ACGCGGAAUACUUCGA-3' $(18,19)$. The cells were transfected with siRNA duplexes using Oligofectamine (Invitrogen) as per the manufacturer's instructions.

Luciferase reporter assay. Standard dual luciferase assay was performed as we have reported (16). pRL-CMV (Promega, Madison, WI, USA) was used as the internal control. The firefly and renilla luciferase activities were measured using the Dual-Luciferase reporter system (Promega) with a model TD-20/20 Luminometer (EG\&G Berthold, Australia). Firefly luciferase activity value was normalized to renilla activity value. Promoter transcription activity is presented as the fold induction of relative luciferase unit (RLU) comparing to basic pGL3 vector control. RLU= values of firefly luciferase unit/values of renilla luciferase unit.

Immunoblotting. The whole cell lysates were prepared with RIPA lysis buffer (Sigma) supplemented with $0.2 \mathrm{mM}$ phenylmethylsulfonyl fluoride and $1 \mathrm{X}$ protease inhibitor cocktail (Sigma). The protein concentration was determined by bicinchoninic acid assay (BCA protein assay kit, Pierce, Rockford, IL, USA). Immunoblotting was performed as described previously with the antigen-antibody complexes were visualized by the enhanced chemiluminescence (ECL) system (Amersham Biosciences, Little Chalfont Buckinghamshire, UK) $(17,19)$.

Preparation of nuclear extract and electrophoretic mobility shift assay (EMSA). The nuclear proteins of cells with different treatments were extracted as reported previously by us (17). Double strand p53 consensus oligonucleotide and XAF1 promoter-specific DNA probes were synthesized. After annealing, DNA probes were labeled with $5 \mathrm{mCi}$ of $\gamma^{-32} \mathrm{P}-$ ATP (PerkinElmer Life and Analytical Sciences) using T4 polynucleotide kinase (Promega). For EMSA, total reaction mixtures containing $10 \mathrm{mM}$ Tris/ $\mathrm{HCl}$ ( $\mathrm{pH} 7.5$ ), $1 \mathrm{mM} \mathrm{MgCl}{ }_{2}$, $0.5 \mathrm{mM}$ DTT, $0.5 \mathrm{mM}$ EDTA, $50 \mathrm{mM} \mathrm{NaCl}, 4 \%$ glycerol and $50 \mu \mathrm{g}$ of poly $(\mathrm{dI}-\mathrm{dC})-$ poly $(\mathrm{dI}-\mathrm{C}) / \mathrm{ml}$, were incubated with $3 \mu \mathrm{g}$ of nuclear extracts and various unlabelled competing oligonucleotides for $10 \mathrm{~min}$ at room temperature, followed by addition of $1 \mu \mathrm{l}$ [(0.5-2)x $10^{5}$ c.p.m.] of the various ${ }^{32} \mathrm{P}$-endlabelled oligonucleotides. Samples were separated by electrophoresis on an $8 \%$ non-denaturing polyacrylamide gel, with detection of radioactive bands by autoradiography for $16-24 \mathrm{~h}$ at $-80^{\circ} \mathrm{C}$.

Site-directed mutagenesis. The QuikChange Site-Directed Mutagenesis kit (Stratagene) was used to generate constructs with mutation of the putative p53-binding elements. The DNA sequences of mutant plasmids were confirmed by gene sequencing. Wild-type and mutant sequence of P1 and P5 with the mutant basepairs underlined were as follows: (P1, wild-type) 5'-AGATCTCCTCCCTCCCTGAAGCTGTGGG CTGGGCCA-3'; (P1, mutant) 5'-AGATCTCCTCCСТCCC GGAATGCGTGGGCTGGGCCA-3'. (P5, wild-type) 5'-CCG AAAGCCTGCAGAGAGCAGAACATGGAAGGAGA-3'; (P5, mutant) 5'-CCGAAAGCCTGCAGAGAGCAGACGCT GGAAGGAGA-3'. The wild-type and mutant pLuc-107 constructs were renamed as pLuc-107-WT, pLuc-107-P1/MT and pLuc-107-P5/MT, respectively.

Statistical analyses. Data are expressed as means \pm SD. Differences in numeric data between groups were analyzed by independent-samples t-test using SPSS 11.0 software. A $\mathrm{p}<0.05$ was considered statistically significant.

\section{Results}

Negative correlation between XAF1 expression and the p53 status of GI cell lines. Byun et al (15) reported that loss or 
A
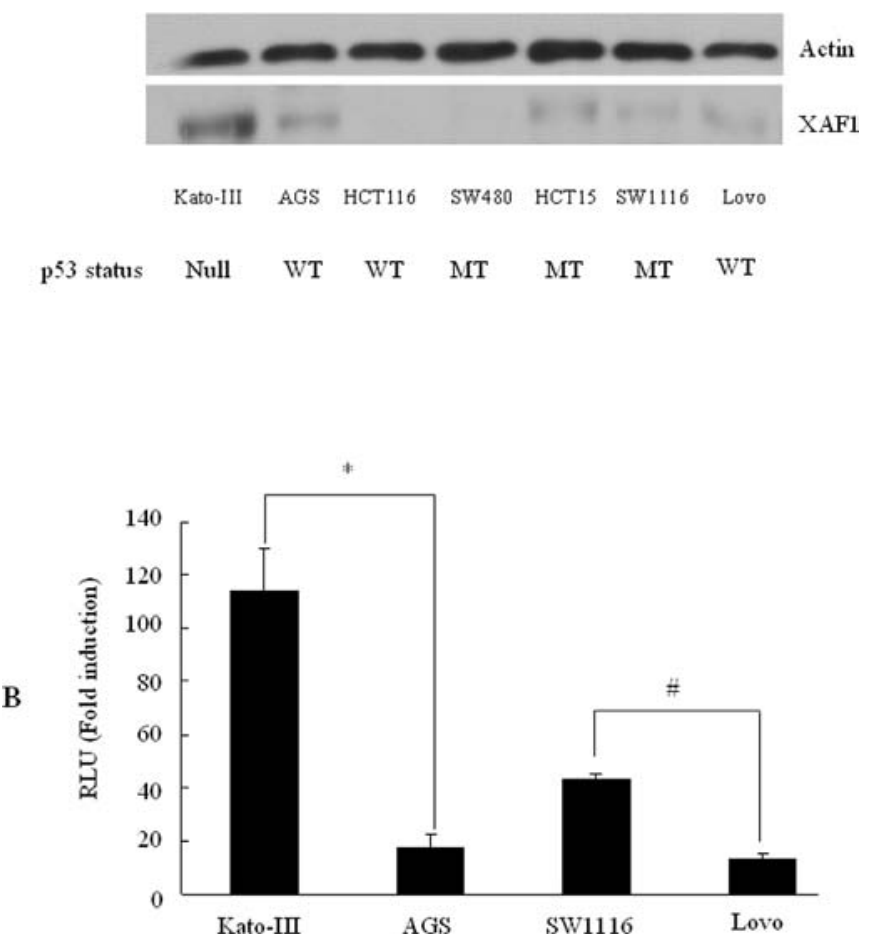

Figure 1. XAF1 expression was negatively correlated with p53 status in GI cancer cell lines. (A) XAF1 expression in GI cancer cell lines with different p53 status was detected by Western blot with actin as the internal control. These figures were the representatives of three experiments with similar results. (B) Transcription activities of the XAF1 promoter construct pLuc107 in different GI cell lines were evaluated by dual luciferase assay. The result was expressed as the fold induction of relative luciferase unit (RLU). ${ }^{*}, \# \mathrm{p}<0.05$. This experiment was repeated for 4 times with similar results.

abnormal reduction of XAF1 expression in GI cancer tissues was inversely correlated with p53 mutations. To confirm their finding, we detected XAF1 expression in 7 GI cancer cell lines with different p53 status (20-24). We found that the highest expression of XAF1 in Kato-III cells, a p53 null gastric cancer cell line (22). On the contrary, the expressions of XAF1 in three cell lines with wild-type p53 including AGS, HCT116 and LoVo were low or absent $(21,23,24)$. $\mathrm{XAF} 1$ expression in p53 mutated cell lines were also low (Fig. 1A).

In addition, we tested the transcription activities of pLuc107 in 4 GI cell lines. We found the fold induction of pLuc107 in Kato-III, AGS, SW1116 and LoVo cells were 113.68ะ $15.93,16.98 \pm 5.46,42.9 \pm 2.27$ and $13.18 \pm 1.85$, respectively (Fig. 1B). Similarly, the activity of XAF1 promoter was high in p53-null cells and low in cells with wild-type p53.

Alteration of $p 53$ expression regulates negatively the XAF1 expression. To directly evaluate the role of $\mathrm{p} 53$ in XAF1 expression, we first ectopically overexpressed wild-type and mutant p53 in Kato-III cells by transiently transfecting the expressing vectors. As shown in Fig. 2A, we found that ectopic overexpression of wild-type but not mutant p53 suppressed XAF1 expression. We then assessed XAF1 promoter activity by sequential transfection with $\mathrm{p} 53$ vectors
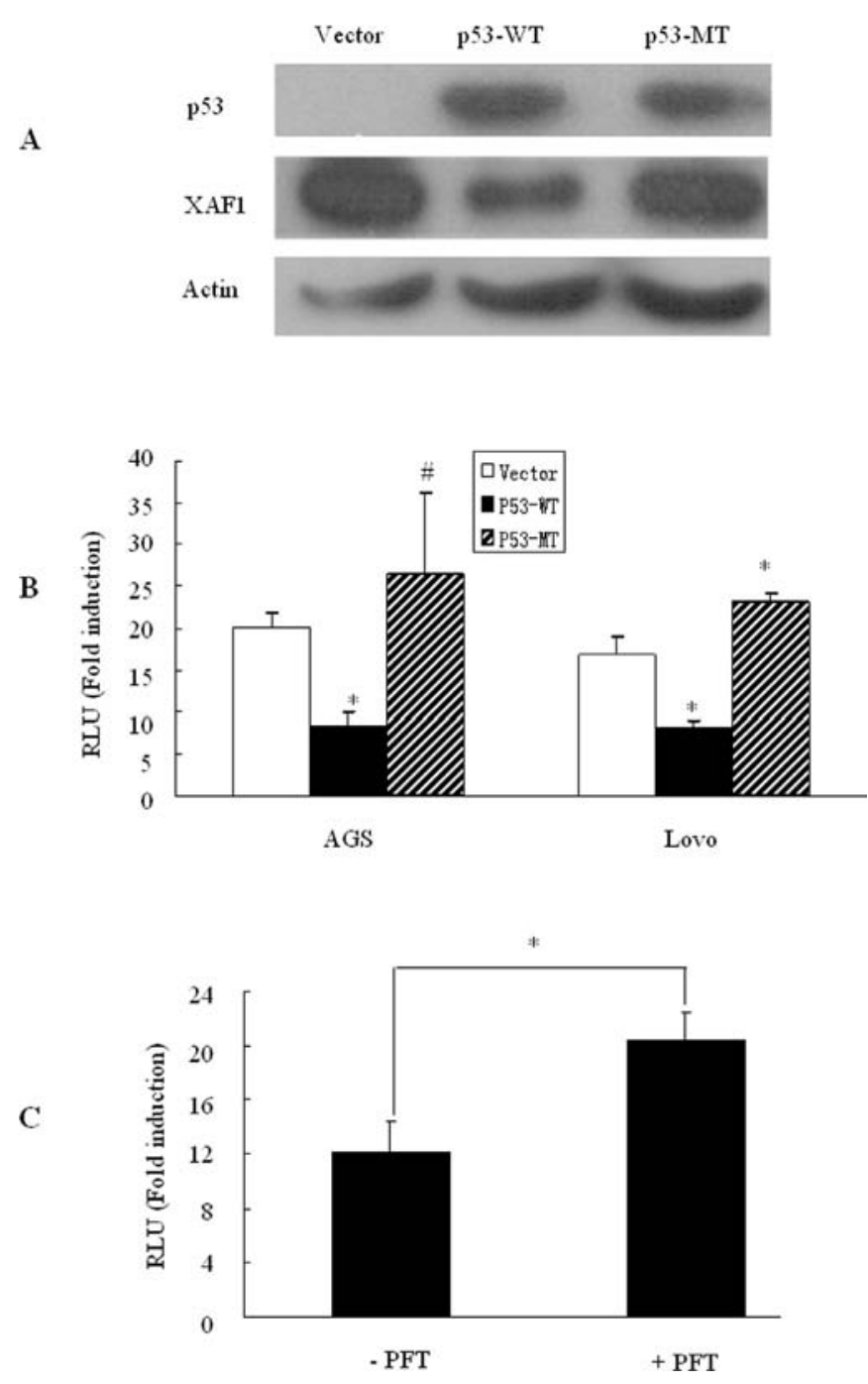

Figure 2. XAF1 expression was negatively modulated by p53. (A) Kato-III cells were transiently transfected with pcMV-tag1 (vector), pCMV-p53 (p53-WT) or pCMV-p53mt135 (p53-MT) for $48 \mathrm{~h}$. XAF1 and p53 expression were detected by Western blot with actin as the internal control. (B) The transcription activities of pLuc-107 in AGS and LoVo cells transfected with vector, wildtype and mutated p53 constructs were assessed by dual luciferase assay. "p $<0.05$ and ${ }^{*} p>0.05$ comparing with the vector controls. (C) The transcription acitvities of pLuc-107 in AGS cells in presence of either vehicle control or pifithrin $\alpha$ as assessed by dual luciferase assay. ${ }^{*} \mathrm{p}<0.05$.

followed by pLuc-107. The transcription activity of pLuc107 in AGS cells transfected with pCMV vector, wild-type p53 and mutant p53 vectors were $20.1 \pm 1.56,8.39 \pm 1.58$ and $26.56 \pm 9.68$, respectively while that in LoVo cells were $16.88 \pm 1.97,8.08 \pm 0.88$ and $23.1 \pm 1.15$, respectively (Fig. 2B). We showed that wild-type p53 significantly suppressed XAF1 promoter activity when comparing with vector control $(\mathrm{p}<0.05)$. Transfection with mutant $\mathrm{p} 53$ increased XAF1 expression in both cell lines, however, significant difference was only found in LoVo $(\mathrm{p}<0.05)$ but not in AGS $(\mathrm{p}>0.05)$ cells.

We suppressed p53 function using a well-defined p53 inhibitor, PFT (25). Fig. $2 \mathrm{C}$ shows that the transcription activities of pLuc-107 were $12.08 \pm 2.24$ and $20.49 \pm 2.01$, 
$\mathbf{A}$

B

$\begin{array}{cccc}\text { P1 } & -107 \text { ATCTCCTCCCTCCCTGAAGCTGT GG } & -83 \\ & & \frac{\mathbf{q} 1}{\mathbf{q} 2} & \\ \text { P2 } & -82 & \text { GCTGGGCCATCGGAAAACT } & -64 \\ \text { P3 } & -63 & \text { TTCAGTTTTGTTTCCTTGCCTGC } & -41 \\ \text { P4 } & -40 & \text { AAGAAACGAAACTCAACCGAA } & -20 \\ \text { P5 } & -19 & \text { AGCCTGCAGAGAGCAGAACATG } & +3 \\ & & \mathbf{q}^{3} & \end{array}$

C

Consensus p53 oligonucleotides (Sense strand) 5-TACAGAACATGTCTAAGCATGCTGGGGACT - -3'

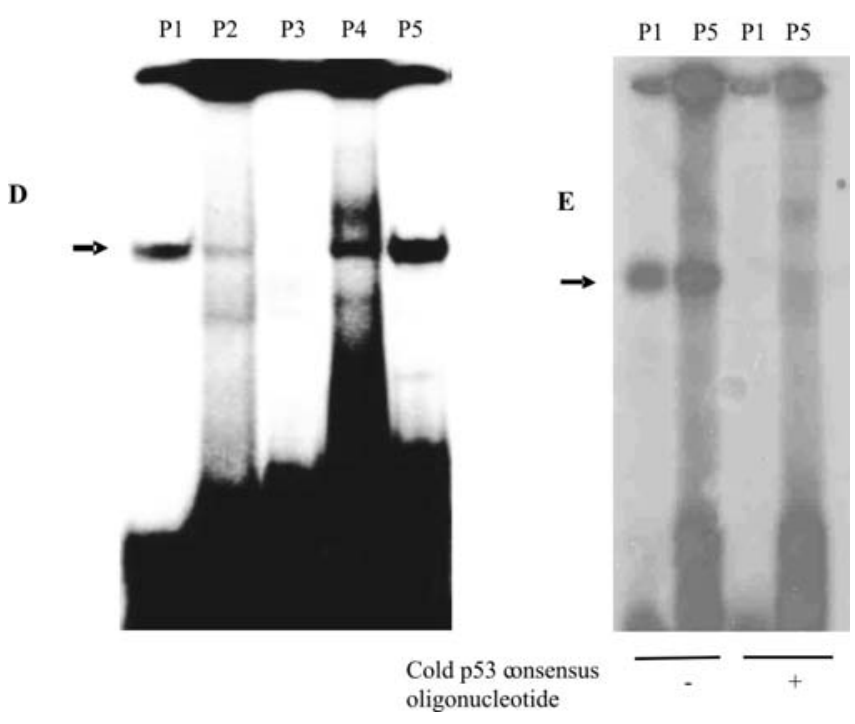

Figure 3. Identification of $\mathrm{p} 53$ binding sequence within XAF1 promoter. (A) Composition of the consensus p53 binding sequence (half-site). (B) Sequence of the five probes for EMSA and their location within XAF1 promoter. The p53 binding quarter-sites (q1, q2 and q3) were underlined. Five to 8 base pairs were overlapped between the two neighboring probes. (C) Sequence of the p53 consensus oligonucleotides. (D) Double strand probes were labeled with ${ }^{32} \mathrm{P}$ and bound to the recombinant $\mathrm{p} 53$ protein with or without pre-incubation with 100-fold excess of unlabelled (cold) p53 consensus oligonucleotide. The binding bands were displayed by EMSA. (E) Binding activities of P1 and P5 to recombinant p53 protein as detected by EMSA.

respectively in the absence or presence of PFT $(\mathrm{p}<0.05)$. Suppression of p53 by chemical inhibitor also stimulated XAF1 transcription.

Identification of the putative p53 binding element within $X A F 1$ promoter. The consensus p53 binding element is composed of two copies of the $10 \mathrm{bp}$ (half-site) motif listed in Fig. 3A $(3,6)$. This half-site can be further divided into two quarter-sites (head and tail quarter-site). pLuc-107 contains $107 \mathrm{bp}$ sequences (including transcription starting site) upstream of the ATG translation starting site. Through searching the putative p53 responsive element (p53RE) using TF search (www.cbrc.jp/research/db/TFSEARCH.html), we

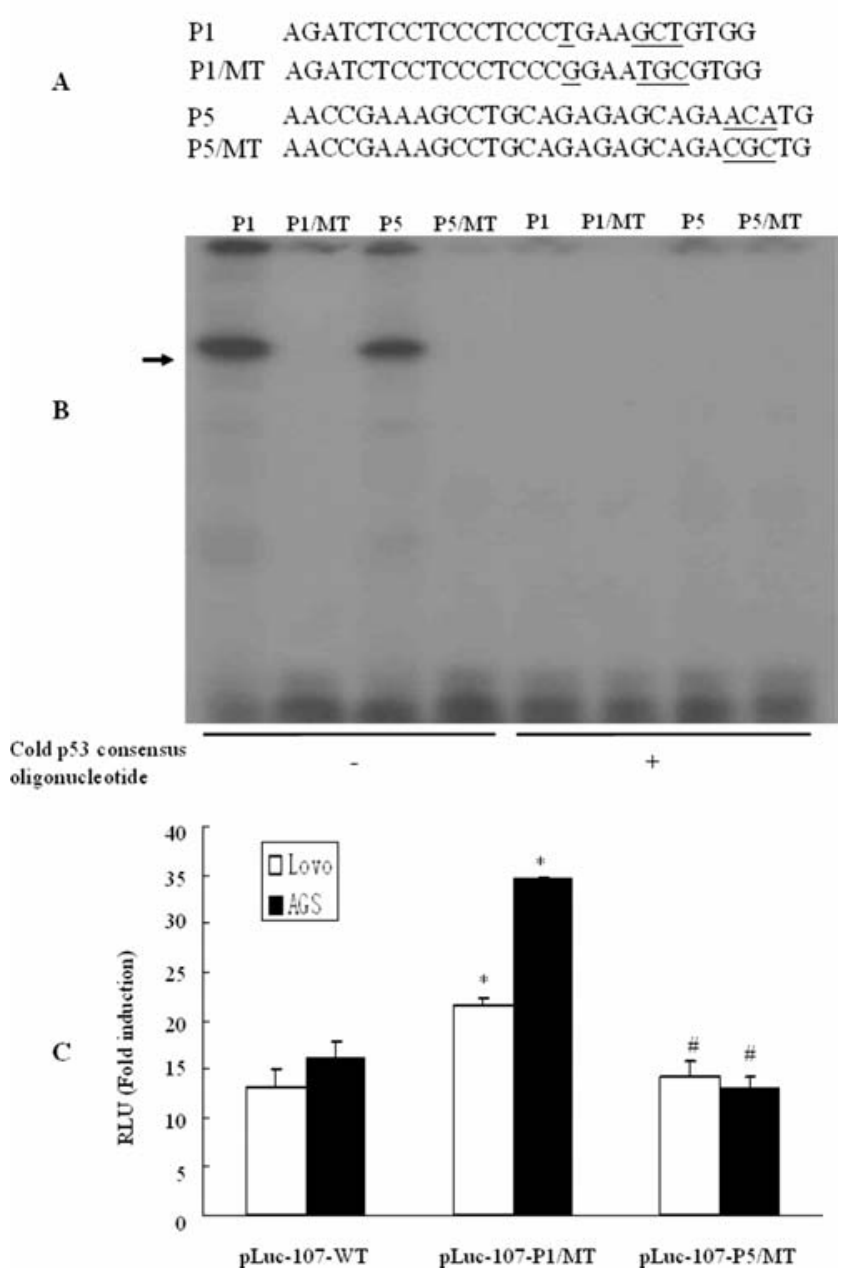

Figure 4. Identification of p53 responsive elements. (A) Sequence of wildtype and site-mutant P1 and P5 probes. (B) Binding activities of the wildtype and mutant (MT) P1 and P5 to the recombinant p53 protein as determined by EMSA. (C) Transcription activities of wildtype (WT), P1 (P1/MT) and P5 (P5/MT) mutant pLuc-107 constructs in LoVo and AGS cells as determined by dual luciferase assay. ${ }^{*} \mathrm{p}<0.05 ;{ }^{*} \mathrm{p}>0.05$ comparing with the wildtype vector.

only found some potential binding elements with their identity to the consensus sequences less than 50\%. However, we were able to find three quarter-sites of $\mathrm{p} 53$ responsive elements, they located at -95 to $-90 \mathrm{nt}$ (q1), -86 to $-90 \mathrm{nt}$ (q2) and -4 to $+1 \mathrm{nt}(\mathrm{q} 3)$, respectively (Fig. 3B). Q1 and q2 were located continuously without any insertion with their configuration being at tail-tail orientation. Thus we considered it as a half-site p53RE (-95 to -86 nt). We then synthesized 5 double strand DNA probes that covered the entire $107 \mathrm{bp}$ segment with 5-8 base pairs of overlapped sequences between the two neighboring probes. The core probe sequences are shown in Fig. 3B. P1 contained p53RE while P5 contained q3. EMSA assay showed that specific binding bands occurred between the recombinant p53 protein with $\mathrm{P} 1$ and P5 (Fig. 3D). Moreover, the binding between P1/P5 and p53 protein could be completely blocked by pre-incubation with excessive nonlabeled double strand p53 consensus oligonucleotides (Fig. 3E). These finding suggested the existence of p53 binding elements within XAF1 promoter. 


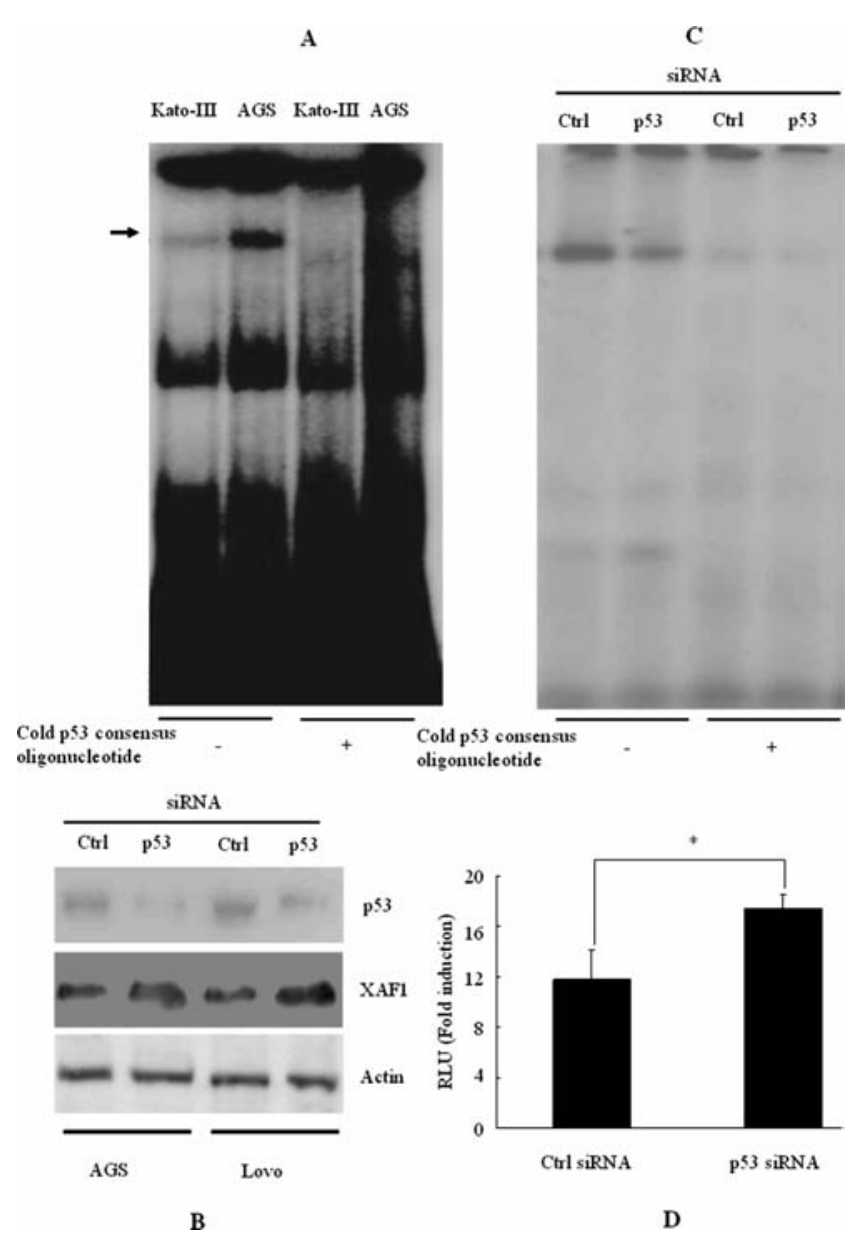

Figure 5. p53 knockdown inhibited the binding activity of its responsive element and increased XAF1 transcription. (A) Three micrograms of the nuclear proteins of AGS and Kato-III cells were incubated with ${ }^{32} \mathrm{P}$ labeled $\mathrm{P} 1$ probe in the presence or absence of excessive cold p53 consensus oligonucleotide. Binding activity was detected by EMSA assay. (B) AGS and LoVo cells were transfected with control or p53 siRNA for $48 \mathrm{~h}$, the expressions of p53 and XAF1 were detected by Western blot with actin as the internal control. (C) AGS cells were transfected with control or p53 siRNAs for $48 \mathrm{~h}$. The nuclear proteins were extracted to detect their binding to labeled P1 probe by EMSA assay. (D) AGS cells were co-transfected with siRNA and pLuc-107 constructs. The transcription activities were assessed by dual luciferase assay. ${ }^{*} \mathrm{p}<0.05$, comparing with the control siRNA.

Identification of the functional p53 responsive element. To define the specific sequence that mediated p53-induced XAF1 suppression, we first synthesized mutant P1 and P5 probes with p53RE and q3 being mutated, respectively. The wild-type and mutant (MT) sequences are listed in Fig. 4A. Similarly, we generated two mutant reporter constructs of pLuc-107 with the same sequences being mutated by site-directed mutagenesis. We first tested the binding activities of mutant probes by EMSA assay. We showed that the mutation of both elements completely abrogated their binding activities to p53 protein (Fig. 4B), indicating the mutant sites were indispensable for binding between $\mathrm{p} 53$ and XAF1 promoter.

We then assessed the effect of site mutation on XAF1 promoter activity. As shown in Fig. 4C, P1 mutation increased the transcriptional activities of pLuc-107 in both AGS and LoVo cell lines. The fold induction of RLU were $13.18 \pm 1.85$, $21.43 \pm 0.94$ and $14.25 \pm 1.49$, respectively for pLuc-107-WT, pLuc-107-P1/MT and pLuc-107-P5/MT, respectively in
LoVo cells. Significant difference was found between pLuc107-WT and pLuc-107-P1/MT $(\mathrm{p}<0.05)$, but no difference was found between pLuc-107-WT and pLuc-107-P5-MT ( $\mathrm{p}>0.05$ ). Similar result was obtained from AGS cells (Fig. 4C). These results indicated the sequence between -95 to $-86 \mathrm{nt}$ was a functional p53RE that mediated p53-induced XAF1 suppression.

p53 knockdown inhibits the binding activity of its responsive element and increases XAF1 transcription. Subsequently, we examined the effect of p53 knockdown on XAF1 expression. We first compared the binding activity of P1 with the nuclear extract of cells with different p53 status. As shown in Fig. 5A, the nuclear extract of AGS (p53 wild-type) (21) but not KatoIII (p53 null) (22) could bind to P1 specifically. Secondly, we suppressed p53 expression by siRNA. We found that p53 knockdown increased XAF1 expression in both AGS and LoVo cells (Fig. 5B). p53 siRNA decreased the binding between P1 and the nuclear extract of AGS as demonstrated by EMSA (Fig. 5C). Finally, we showed that co-transfection of p53 siRNA increased the transcription of XAF1 promoter pLuc-107 in AGS cells (Fig. 5D). The RLU fold induction of control and p53 siRNA was $11.8 \pm 2.39$ and $17.47 \pm 1.06$, respectively. Significant difference was found between the two siRNAs.

\section{Discussion}

In this study, we defined an exclusive mechanism between two tumor suppressors, p53 and XAF1 gene. A p53 responsive element (-86 to $-95 \mathrm{nt}$ ) was identified within the core promoter of XAF1 gene. p53 protein bound to this element with high affinity. Abrogation of this element by site-mutation or p53 knockdown inhibited the binding and abolished the inhibiting effect of p53 on XAF1 transcription.

The negative correlation between XAF1 and p53 was first reported by Byun et al (15). During the investigation of methylation-induced gene silencing of XAF1 in gastric cancer tissues, they found loss or down-regulation of XAF1 expression presented in 4 of $5(80 \%)$ cell lines with wild-type p53, but only in 2 of 10 (20\%) cell lines harboring homozygous deletions or mutations of p53. In addition, 7 of 9 (78\%) primary tumors with reduced XAF1 expression carried wild-type p53 and 13 of 15 (87\%) primary tumors with mutant p53 showed normal expression of XAF1. In this study, we used different cell lines. Our results were generally consistent with their finding that wild-type p53 suppressed XAF1 expression. Mutant p53 did not suppress XAF1 expression and even had some stimulating effect on XAF1 promoter activity in LoVo cells (Fig. 2B). This effect might be caused by abrogation of the interaction between the endogenous wild-type p53 protein and its binding element by ectopically overexpressed mutant p53 protein in LoVo cells.

p53 protein exists in cells with damaged DNA (transformed cell) as a tetramer (5-7). Each subunit of such a tetramer binds to one quarter-site of p53RE. Thus, it was conventionally believed that four quarter-sites of p53RE were all required for p53 binding (6-9). Most of p53REs identified so far are composed of two half-sites (3). This might be the reason that we could not find a typical p53RE through TFsearch. 
However, we found only one half-site of p53RE within XAF1 promoter (-95 to $-86 \mathrm{nt})$. EMSA assay confirmed its high affinity to both recombinant $\mathrm{p} 53$ protein and nuclear extract of cells with wild-type p53, while site mutation confirmed the specificity of its binding activity and function. Some other p53 target genes such as PTK2 (FAK) had also only one functional half-site of p53RE (AAGCAAGC). Interestingly, p53 also functioned as a repressor for FAK transcription (26). Our finding was further supported by two recent structural studies in which it was reported that the traditionally believed mode with four p53 monomers binding at all four DNA quarter-sites did not cause linear DNA to bend. Alternatively, p53 tetramer could use only two monomers to recognize DNA sequence and induce DNA bending $(9,10)$. Consistent with these reports, we found that one quarter-site (P5) of p53RE could bind to recombinant p53 but had no function, while the binding to two quarter-sites (P1) was effective in exerting biological function as determined by mutation experiments (Fig. 4C).

Not surprisingly, the p53RE we identified is not $100 \%$ identical to that of the consensus sequence. The last base $(\mathrm{G})$ of p53RE within XAF1 prompter (CCTGAAGCTG) was mismatched. The identities between the p53RE of the 129 p53 target genes and the consensus sequence were from 42.33 to $93.98 \%$ (3). Although p53 predominantly functions as a tumor suppressor, its target genes can be either the tumor suppressor or oncogenes. In addition to suppressing the oncogenic genes such as survivin and to activate pro-apoptotic genes such as puma and bax, p53 can also activate oncogenic genes EGF-R and H-RAS. Our finding suggested that p53 could even suppress a tumor suppressor gene.

We had previously reported that XAF1 was a stressexclusive protein which was negatively modulated by heat shock factor 1 (HSF1) (27). Interestingly, HSF1 was closely correlated with p53 in exerting their functions. Both proteins are stress-activated proteins and play synergized effect in carcinogenesis (28). p53 inhibitor PFT also suppressed the functions of HSF1 (29). Moreover, HSF1 was required for the nuclear translocation and the subsequent transaction function of p53 (30). Therefore, our current finding that p53 suppressed XAF1 transcription supported the existence of a self-protective mechanism for cells with damaged DNA or in response to stress stimulation. Lack of XAF1 expression in cells with wild-type p53 can prevent cells from undergoing redundant apoptosis, while p53 deficient cells expressing high level of XAF1 can sensitize the cells to apoptosis. This may serve as the second line of eradication mechanism of transformed cells. Nevertheless, the functional correlation between XAF1 and p53 require further investigation.

In conclusion, we identified a p53 responsive element within XAF1 promoter located at -95 to $-86 \mathrm{nt}$ upstream the translation starting codon. This area is contained by the core promoter of XAF1 (16,27). p53 inhibits XAF1 transcription through interaction with this element. Our result implicated XAF1 as a novel p53 target gene with the functional correlation to be elucidated.

\section{Acknowledgement}

This study is supported by the Dean's research fund of Nanfang Hospital, the Southern Medical University.

\section{References}

1. ez-Lazaro M, Fernandez-Gomez FJ and Jordán J: p53: twenty five years understanding the mechanism of genome protection. $\mathrm{J}$ Physiol Biochem 60: 287-307, 2004.

2. Amundson SA, Myers TG and Fornace AJ Jr: Roles for p53 in growth arrest and apoptosis: putting on the brakes after genotoxic stress. Oncogene 17: 3287-3299, 1998.

3. Riley T, Sontag E, Chen P and Levine A: Transcriptional control of human p53-regulated genes. Nat Rev Mol Cell Biol 9: 402-412, 2008.

4. Morrin M, Kelly M, Barrett N and Delaney P: Mutations of Kiras and p53 genes in colorectal cancer and their prognostic significance. Gut 35: 1627-1631, 1994.

5. Wei CL, Wu Q, Vega VB, et al: A global map of p53 transcription-factor binding sites in the human genome. Cell 124: 207-219, 2006.

6. El-Deiry WS, Kern SE, Pietenpol JA, Kinzler KW and Vogelstein B: Definition of a consensus binding site for $\mathrm{p} 53$. Nat Genet 1: 45-49, 1992.

7. Janz C, Susse S and Wiesmuller L: p53 and recombination intermediates: role of tetramerization at DNA junctions in complex formation and exonucleolytic degradation. Oncogene 21: 2130-2140, 2002

8. Cho Y, Gorina S, Jeffrey PD and Pavletich NP: Crystal structure of a p53 tumor suppressor-DNA complex: understanding tumorigenic mutations. Science 265: 346-355, 1994.

9. Ma B, Pan Y, Zheng J, Levine AJ and Nussinov R: Sequence analysis of p53 response-elements suggests multiple binding modes of the p53 tetramer to DNA targets. Nucleic Acids Res 35: 2986-3001, 2007

10. Ma B and Levine AJ: Probing potential binding modes of the p53 tetramer to DNA based on the symmetries encoded in p53 response elements. Nucleic Acids Res 35: 7733-7747, 2007.

11. Liu X, Miller CW, Koeffler PH and Berk AJ: The p53 activation domain binds the TATA box-binding polypeptide in Holo-TFIID, and a neighboring p53 domain inhibits transcription. Mol Cell Biol 13: 3291-3300, 1993.

12. Shiio $Y$, Yamamoto $T$ and Yamaguchi N: Identification of a DNA element that can enhance p53-mediated transactivation. Oncogene 8: 2059-2065, 1993.

13. Liston P, Fong WG, Kelly NL, et al: Identification of XAF1 as an antagonist of XIAP anti-Caspase activity. Nat Cell Biol 3: 128-133, 2001.

14. Fong WG, Liston P, Rajcan-Separovic E, St Jean M, Craig C and Korneluk RG: Expression and genetic analysis of XIAPassociated factor 1 (XAF1) in cancer cell lines. Genomics 70: 113-122, 2000.

15. Byun DS, Cho K, Ryu BK, Lee MG, Kang MJ, Kim HR and Chi SG: Hypermethylation of XIAP-associated factor 1, a putative tumor suppressor gene from the $17 \mathrm{p} 13.2$ locus, in human gastric adenocarcinomas. Cancer Res 63: 7068-7075, 2003.

16. Zou B, Chim CS, Zeng H, et al: Correlation between the singlesite CpG methylation and expression silencing of the XAF1 gene in human gastric and colon cancers. Gastroenterology 131: 1835-1843, 2006.

17. Wang J, Peng Y, Sun YW, et al: All-trans retinoic acid induces XAF1 expression through an interferon regulatory factor-1 element in colon cancer. Gastroenterology 130: 747 758, 2006.

18. Brummelkamp TR, Bernards R and Agami R: A system for stable expression of short interfering RNAs in mammalian cells. Science 296: 550-553, 2002

19. Wang J, Yang Y, Xia HH, et al: Suppression of FHL2 expression induces cell differentiation and inhibits gastric and colon carcinogenesis. Gastroenterology 132: 1066-1076, 2007.

20. Liu L, Nakatsuru Y and Gerson SL: Base excision repair as a therapeutic target in colon cancer. Clin Cancer Res 8: 2985-2991, 2002.

21. Jiang XH, Wong BC, Lin MC, et al: Functional p53 is required for triptolide-induced apoptosis and $\mathrm{AP}-1$ and nuclear factor-kappaB activation in gastric cancer cells. Oncogene 20: 8009-8018, 2001.

22. Osaki M, Tatebe S, Goto A, Hayashi H, Oshimura M and Ito H: 5-Fluorouracil (5-FU) induced apoptosis in gastric cancer cell lines: role of the p53 gene. Apoptosis 2: 221-226, 1997.

23. Toscano F, Fajoui ZE, Gay F, et al: p53-mediated upregulation of DcR1 impairs oxaliplatin/TRAIL-induced synergistic antitumor potential in colon cancer cells. Oncogene 27: 4161-4171, 2008. 
24. Yu LF, Wang J, Zou B, et al: XAF1 mediates apoptosis through an extracellular signal-regulated kinase pathway in colon cancer. Cancer 109: 1996-2003, 2007.

25. Komarov PG, Komarova EA, Kondratov RV, Christov Tselkov K, Coon JS, Chernov MV and Gudkov AV: A chemical inhibitor of p53 that protects mice from the side effects of cancer therapy. Science 285: 1733-1737, 1999.

26. Golubovskaya V, Kaur A and Cance W: Cloning and characterization of the promoter region of human focal adhesion kinase gene: nuclear factor kappa B and p53 binding sites. Biochim Biophys Acta 1678: 111-125, 2004.

27. Wang J, He H, Yu L, et al: HSF1 down-regulates XAF through transcriptional regulation. J Biol Chem 281: 2451-2459, 2006
28. Dai C, Whitesell L, Rogers AB and Lindquist S: Heat shock factor 1 is a powerful multifaceted modifier of carcinogenesis. Cell 130: 1005-1018, 2007.

29. Komarova EA, Neznanov N, Komarov PG, Chernov MV, Wang $\mathrm{K}$ and Gudkov AV: p53 inhibitor pifithrin alpha can suppress heat shock and glucocorticoid signaling pathways. J Biol Chem 278: 15465-15468, 2003.

30. Li Q, Feldman RA, Radhakrishnan VM, Carey S and Martinez JD: Hsf1 is required for the nuclear translocation of p53 tumor suppressor. Neoplasia 10: 1138-1145, 2008. 